\title{
Fast Sonochemical Exfoliation of Hematene type Sheets and Flakes from Hematite Nanoarchitectures Shows Enhanced Photocurrent Density
}

\author{
Purvika Agarwal ${ }^{a}$, Debajeet K. Bora ${ }^{a^{*} 1}$ \\ Centre for Nano and Material Sciences, JAIN (Deemed - to - be University), Jain Global Campus, \\ Bangalore 562112, India
}

\section{Abstract}

This report demonstrates a sonochemical exfoliation strategy to form a hematene nanosheet and flakes from hematite nanopowder and thin film. The optical properties of obtained hematene sheets and flakes show unusual features that differ from that of a pristine Hematite film, which allows it to further apply for photoelectrochemical functionality enhancement. Here, we found that the ultrasonicated Hematite film with Hematene flakes shows enhanced photocurrent density than the pristine Hematite film. The increased light absorption property of the Hematene flakes due to the scattering effect helps in achieving it. The exfoliation process is further confirmed by the X-ray diffraction of the Hematene sheet where (104) plane Bragg peak

is split up after the ultrasonication process. It is corroborated with a qualitative predictive mechanism for the fast exfoliation of nanosheets and flakes. Here, surface energies and hydroxylation of crystal facets are proposed to be the factor responsible for the quick exfoliation process.

\footnotetext{
${ }^{1}$ corresponding author: Debajeet K. Bora, Ph.D. E-Mail: debajeet.bora@jainuniversity.ac.in, debajeet1@outlook.com
} 


\section{Introduction}

22 The emergence of 2D materials by exfoliation techniques finds paramount importance in the current

23 development of new semiconductors. In this regard, both graphene and metal chalcogenides are 24 studied and applied broadly. The first scotch tape-based exfoliation of 2D materials from carbon25 based allotrope Graphite leads to the discovery of Graphene, and it pushes a new regime of materials 26 research into tremendous applications, primarily for semiconductor devices and quantum physics 27 study [1]. Next, the liquid-based exfoliation of layered materials provides high surface area results 28 in enhanced optical and electrochemical properties, and it is possible to exfoliate them into 29 nanosheets due to the weak out-of-plane bonding [2]. The chemical exfoliation of metal carbides 30 forms 2D transition metal carbide layers known as Mxene, and it finds various applications from 31 electromagnetic shielding to energy conversion and storage application [3]. Other 2D materials such 32 as phosphorene, transition metal chalcogenides are also well studied for their diverse application [4 33 - 8]. Besides these, recently, interest rises to develop 2D metallene nanomaterials such as silicene, 34 antimonene, germanene, stanene [9]. Within this category, the single layer of PdTe made by 35 electrostatic doping shows polar metal characteristics and proven by first-principle calculations 36 [10]. These materials are Van der Waals solids due to the force existing in between the interlayers 37 in bulk material, as observed in the case of Graphene [11].

38 Metal oxides based 2D materials still need further research to decipher their role in various applications. Several metal oxides, such as $\mathrm{TiO}_{2}, \mathrm{WO}_{3}, \mathrm{ZnO}$ and $\mathrm{Co}_{3} \mathrm{O}_{4}$, have been rigorously studied and converted to their two-Dimensional forms in the form of layered double hydroxide and

41 shows many different electrochemical properties [12]. A recent candidate of 2D copper (II) oxide nanosheet [13] is prepared by the surface Sulphur functionalization, which shows semiconductor43 conductor transition. Besides these, Iron oxides and their analogues also possess widespread 44 applications in the diverse fields of catalysis [14], magnetic storage media [15], gas sensing, 
Lithium-ion batteries [16] and other biological applications [17]. Hematite $\left(\alpha-\mathrm{Fe}_{2} \mathrm{O}_{3}\right)$, an n-type semiconductor, possesses excellent stability and an appropriate bandgap, making it well known for

47 solar water splitting photosynthesis application [18]. However, iron oxide-based 2D materials 48 such as layered double hydroxide, which is generally not photoactive, are used in electrocatalytic 49 hydrogen production due to the high surface area, low overpotential and fast water oxidation 50 kinetics. It shows a higher catalytic turnover process for the oxygen evolution reaction [19]. The 51 photoactive 2D exfoliated layers of the Hematite semiconductor has not been explored. With the 52 discovery of the ultrasonication method, various nanostructured materials with reasonable control 53 over size, morphology and crystallographic structure can be formed. Cavitation and Nebulization 54 are primary physical phenomena associated with the ultrasonic synthesis of nanomaterials [20]. 55 Applying it for the exfoliation of the 2D single layer from metal oxides into sheets and flakes [21] 56 is an exciting area of materials development. Balan et al. reported a sonochemical route for the 57 exfoliation of 2D Hematite from geological Iron Ore, called Hematene. The Hematene layer forms 58 by breaking covalent bonds between the Fe-O layers of Hematite crystal by the ultrasonication 59 process [22]. They used the sheet-like layer for the $\mathrm{TiO}_{2}$ photoelectrode sensitization to measure 60 the photocurrent density with an application toward water-splitting mediated hydrogen evolution. 61 The Hematene obtained also shows ferromagnetic order, while Hematite usually shows 62 antiferromagnetic behaviour. Hematene is a non-Van der waal material, and it shows exotic 63 magnetic properties with a magnetic moment of $4 \mu_{\mathrm{B}}$ per atom according to a recent DFT study 64 followed by its surface functionalization on Stanene converts Hematene into ferrimagnets [23]. 65 Hematene, as a non-van der Waal 2D material, is also chemically stable in the ambient conditions 66 due to its oxygen passivation [24], and next, Hematene based heterostructure $\left(\mathrm{Hematene} / \mathrm{BaTiO}_{3}\right)$ 67 is also developed to tune the magnetic properties [25]. Not only magnetism but Hematene also find 68 suitable application in the biomedical sector and associated energy research with the development 
of it for the combined application of glucose sensing and electrocatalytic action followed by the 70 development of a biofuel cell operated by the glucose [26]. For the oxygen evolution 71 electrocatalysis study, recently defect enriched Hematene has a current density of $10 \mathrm{~mA} / \mathrm{cm}^{2}$ at $720.250 \mathrm{~V}$ overpotential [27]. The energy storage outlook of this material is also recently taken into 73 consideration and is published elsewhere [28].

74 Hematene sheets are applied for solar water splitting reactions. However, the direct application of 75 Hematene flakes on a pristine hematite photoanode for photoelectrochemical functionality 76 enhancement is not carried out so far. The motivation of the current study is the ultrasonic treatment 77 of Hematite film to get Hematene sheets and flakes and to understand its effect on photocurrent 78 density enhancement to fill this gap. The next question is whether a short sonochemical period is 79 enough to make such kinds of nanosheets? As the usual exfoliation time in the studies mentioned 80 above, geological ores are long as the Fe-O strong bonding will not efficiently allow the exfoliation 81 process [22]. However, exfoliation of Hematene from Hematite nanoparticle and their thin film can 82 be an additional advantage concerning surface energies of different crystalline facts within different 83 morphological variants of iron oxide [29]. Hence, the development of a fast exfoliation method is 84 another motivation of the current study.

\section{Methods}

\subsection{Materials}

87 All chemicals used in the current study have been procured from commercial sources and possessed 88 97-99\% purity. Ferric (III) Chloride hexahydrate $\left(\mathrm{FeCl}_{3} \cdot 6 \mathrm{H}_{2} \mathrm{O}\right)$ and Urea $\left(\mathrm{NH}_{2} \mathrm{CONH}_{2}\right)$ was 89 purchased from Sigma-Aldrich. Absolute Ethanol used for washing the ochre yellow precipitate 90 was of analytical grade and was obtained from

91 Changshu (C.S.), while distilled water was distilled in our laboratory. For the deposition of 92 Hematite, FTO glass slides were obtained from Ants Ceramic Limited, Mumbai, India. 


\subsubsection{Synthesis of Hematite nanoparticle and thin film:}

A synthetic protocol similar to previously reported literature has been employed to synthesize the Hematite thin film using water as a solvent $[30,51]$. Here, $1 \mathrm{~g}$ of Ferric Chloride Hexahydrate and $2.2 \mathrm{~g}$ of Urea were mixed with distilled water in a round bottom flask and was stirred at $80^{\circ} \mathrm{C}$. The resultant mixture forms an ochre yellow colour solution after 6 hours of the reaction. The solution was then centrifuged and washed with Ethanol. The residue obtained was dried at $70^{\circ} \mathrm{C}$. Later, this mass was calcinated in the air at $500^{\circ} \mathrm{C}$ for 3 hours to get a reddish-brown colour powder, which is the characteristics colour of Hematite. FTO (Fluorine doped tin oxide) substrate was clean with isopropanol; soap water followed upon by dip-coated in the supernatant solution to obtain thin films of the same. It was then dried and further heat-treated at $500^{\circ} \mathrm{C}$ for 3 hours to get the Hematite film. The thickness of the hematite film is shown in Figure S3, and it is $0.95 \mu \mathrm{m}$. The RMS roughness of the hematite film before sonication is found to be $7.6 \mathrm{~nm}$. We have used the centrifugation process in the isolation of akageneite powders. We have also used the centrifuged process in the isolation and calculation of sonicated Hematene sheet yield. It is a new amendment, as described below.

\subsubsection{Synthesis of Hematene Sheets and Flakes containing Hematite Films}

For the synthesis of Hematene sheets, Hematite nanopowders obtained are ultrasonicated for 5 min in Ethanol in a glass vial. The obtained solution is further applied for TEM characterization to confirm the presence of Hematene. The Hematene flake is formed by the ultrasonication of as obtained Hematite film for $5 \mathrm{~min}$ in Ethanol. Films are taken out and characterized further with FESEM to confirm the presence of Hematene flakes.

The exfoliation yield of the process is calculated by following the protocol as described elsewhere [ 52]. Here, the weight of the empty centrifugation tube is first taken. Then the Hematene sheet solution is centrifuged for $10 \mathrm{~min}$ at a speed of $10000 \mathrm{rpm} / \mathrm{hr}$. After this, the weight of the Hematene 
sheet along with the tube is retaken. The difference comes out as $1 \mathrm{mg}$, which is a low exfoliation 117 yield.

2.2. Characterization of obtained powder and thin films: Hematite nanoparticle's morphology and lattice imaging are studied with high-resolution transmission electron microscopy (model: Thermo Scientific Talos F200S scanning/transmission electron microscope (S / TEM). The TEM sample preparation was done with nanoparticle dispersion in Ethanol with an ultrasonication for 5 min followed by drop cast on a TEM grid. They were then drying it to evaporate the Ethanol in an Eppendorf tube. This result in Hematene sheets like morphology is further studied with the same TEM model with higher resolution. The FESEM and XRD of Hematite nanoparticles are studied with Field Emission Scanning Electron Microscopy (FESEM) JEOL model JSM7100F, equipped with energy dispersive X-rays (EDX) spectrometer operating at $15 \mathrm{keV}$ and Powder X-Ray diffractometer (Rigaku, Ultima IV X-Ray Diffractometer, $\mathrm{Cu} \mathrm{K} \alpha$ radiation). The morphology and EDX mapping of the same is carried out with FESEM (same model). The optical properties of Hematite nanoparticles and thin films are studied with UV-Vis spectroscopy (Shimadzu UV-1800 UV-Vis spectrometer). The surface topography of thin films was investigated on Atomic Force Microscopy (Agilent Technologies, model 5000) in tapping mode. The phase compositions of the thin films were studied on X-ray diffractometer (XRD) Rigaku, Smartlab XRay Diffractometer model with $\mathrm{CuK} \alpha$ radiation of wavelength $1.54 \AA$ in the $2 \theta$ range of 5 to $80^{\circ}$ in parallel beam (grazing incidence) mode. The Reflectance spectra corresponding to thin films were obtained on a UV-VIS-NIR/DRS instrument Perkin Elmer, Lambda 750 in the range 190-700 nm. The emission spectra of thin films were procured from a spectrofluorometer (Horiba Jobin Yvon, FL-1039/40), with $50 \mathrm{~W}$ ozone free Xe source and double Czerny Turner optics having $1200 \mathrm{~g} / \mathrm{mm}$ rating blazed at $500 \mathrm{~nm}$. The XPS spectra were recorded by an ESCA, Kratos Analytical, Axis Supra, U.K. spectrometer equipped with AlK $\alpha 600 \mathrm{~W}, \mathrm{X}$-ray source. The analysis software used here is Image $\mathrm{J}$ 
(Fiji TEM package) for HRTEM FFT, FESEM analysis, Dwyydion for AFM data analysis, Casa 141 XPS for XPS core level data analysis.

142 2.2.1. Photoelectrochemical characterization. The photocurrent density of Hematite film before and 143 after ultrasonication was measured in an Electrochemical Workstation from C.H. instruments, 144 CHI660D Potentiostat model with a Potential range of -10 to $10 \mathrm{~V}$ and Current range of $250 \mathrm{~mA}$. 145 The measurement setup consists of the photoelectrochemical cell (custom built from Borosil) 146 beaker having a 3 -electrode system configuration with $1 \mathrm{MKOH}(\mathrm{pH}=13.6)$ as the electrolyte. $147 \mathrm{Ag} / \mathrm{AgCl}$ on sat. $\mathrm{KCl}$ and platinum wire were used as the reference and counter electrode, 148 respectively. A LED lamp (100mW) from Tec. Inc. USA [tec light] with embedded electronic 149 shutter option was used as the light source to measure the photocurrent density. The transient 150 photocurrent density is taken using the same light source using the built-in light on-off shutter 151 option with both fast and slow interval modes.

\section{3. Results and discussion}

153 Before discussing Hematene sheets and flakes formation, we will first explain the force hydrolysis 154 reaction to form hematite nanoparticles and films. Here, the forced hydrolysis is defined as a reaction of iron trichloride with water [Figure 1- I], and this yields many different morphologies. In this case, we have also used Urea, whose role is to control nanoparticles' shape. Due to an acidic bi-product (carbonic acid) formation, Urea mainly controls nanoparticle morphology, as established in a separate study. It adsorbed on the specific crystal planes of growing nanocrystals [30]. From the SEM characterization of the as-prepared Hematite powders [Figure 1- II], we found that the morphology shows a rod-like pattern, which is a characteristic of the formation of akageneite rods on top of which Hematite nanoparticle nucleates [31]. The heat treatment at $500^{\circ} \mathrm{C}$ results in spherical shape nanoparticles. A similar morphological transition and its growth have been reported 
in earlier literature [32]. The mean nanoparticle size of rod-shaped particles found here is $34 \mathrm{~nm}$, while hematite nanoparticles are $115 \mathrm{~nm}$ [Figure S5]. Next, from the XRD of Hematite nanoparticles, it is found that (104) Bragg planes have the maximum XRD peak intensity than that of (110) plane reveals it as the most exposed crystallographic plane [33]. The XRD patterns of the sample in their powder form have been illustrated in Figure 1-II C and matched well with the JCPDS no. 34-1266, which is usually indexed for akageneite $(\beta-\mathrm{FeOOH})$ phase and, upon thermal treatment of it at $500^{\circ} \mathrm{C}$, undergoes a phase transformation into Hematite $\left(\alpha-\mathrm{Fe}_{2} \mathrm{O}_{3}\right)$. The two prominent peaks (104) and (110), indexed to the JCPDS no. 33-0664, grow from (004) and (11-2) of the Akageneite XRD pattern (before calcination). [33].

\subsection{Sonochemical Exfoliation of Hematene sheets and flakes}

The sonochemical exfoliation process has been carried out for both Hematite nanoparticle powders and the thin film to get Hematene sheets and flakes, as shown in Figure 1-III in a one-pot setup. We called it ultrasonic or sonochemical exfoliation because we observed such nano sheet-like materials after the ultrasonic preparation of nanopowder and film. On ultrasonication for 5 minutes in structural and morphological characteristics. Afterwards, we will discuss the result obtained from the FESEM investigation of the Hematene flakes. Whether these materials possess any characteristics for photoelectrochemical functionality, we have further studied its detailed 
structural, topological, morphological, optical and surface composition investigation with advanced microscopy as well as spectroscopic and diffraction tools.

\subsection{Detail atomic structure investigation of Hematene sheet with HRTEM}

189 We have performed the HRTEM (High-resolution transmission electron microscopy) investigation of ultrasonically dispersed nanoparticles [Figure 2A] to probe the morphologies. Nanoparticles show an aggregate like feature resembling cauliflower in the first observation. Next, we have found a partially hexagonal sheet-like morphology at a $20 \mathrm{~nm}$ scale, which on close inspection at the higher resolution, shows the lattice structure of Hematite [Figure $2 \mathrm{~B}-\mathrm{E}$ ]. Comparing this morphology with the existing literature report [20], the terminology of Hematene comes from a similar lattice structure observed during the ultrasonic exfoliation of naturally available iron ores [20]. The atomic structure of the Hematene sheet formed is further investigated by lattice imaging of Hematene sheet edges, as shown in Figure 2F. Here, the interlayer spacing between two atomic planes of Hematene is $2.66 \AA$, corresponding to the (104) plane of Hematite crystallographic structure. The FFT (Fast Fourier Transform) of HRTEM lattice imaging results in the electron diffraction patterns of hexagonal symmetry, as shown in Figure 2G. A similar hexagonal symmetry is also observed for Hematene obtained by Balan et al. [22]. However, we do not have here direct evidence about the crystallographic projection to know the basal planes. Whereas in other studies, 203 it is the (001) plane [22].

204 The indexing of diffraction spots reveal (131) planes with $\mathrm{d}$ - spacing value closely matched to the JCPDS pattern of Hematite with the rhombohedral crystal system. These observations indicate the crystallographic origin of Hematene nanosheet exfoliated from Hematite lattice with the ultrasonication procedure, and the formation of a free-standing 2-D Hematene sheet is well

208 confirmed. We have made another exciting observation by looking at different edges (yellow 
rectangular portion) of the Hematene sheet lattice fringes. Here, we have obtained a superlattice 210 arrangement of the atomic assembly for the nanocrystallites, as shown in Figure $2 \mathrm{H}$. The d-spacing 211 matches well with (012) Bragg planes, and the plane at a $75^{\circ}$ angle is (104). A closer look at it 212 reveals an evolutionary pattern of the atomic arrangement in a hexagonal order [ Figure 2I]. There 213 are two types of atomic layers observed. The inward layer and outer layers are repeating in ordered 214 manners refer to the formation of a single layer Hematene sheet [22, 27].

215 Nevertheless, we strictly not confirmed it by molecular dynamic simulation as described elsewhere 216 [22], which shows a thickness of $20 \AA$. The FFT of the corresponding lattice fringes [Figure 2J] 217 also indicates the formation of hexagonal spots corresponding to each atom constituting the 218 Hematene crystallographic structure and repeating in a similar pattern. The FFT diffraction spot 219 corresponds to the (131) planes of Hematite. A similar atomic arrangement is observed for the 220 Hematite nanocrystal with a bitruncated dodecahedron shape [29].

221 Next, we have performed the HRTEM of nanoparticle clusters in different ROI (region of interest) 222 with the selected area electron diffraction pattern (SAED) to get further details about the atomic 223 arrangement of hematite nanocrystallites Hematene is exfoliated. The TEM of Hematite 224 nanoparticles (Figure $2 \mathrm{~K} 1$ ) again shows aggregate like features, which offer usual lattice fringes 225 (Figure $2 \mathrm{~K} 2$ ), proves its crystalline nature. The selected area diffraction pattern (SAED) of the 226 crystallites and its analysis (Figure $2 \mathrm{~L}$ and $\mathrm{M}$ ) with Fiji TEM package of Image $\mathrm{J}$ shows ring 227 patterns, where ring numbers 4 and 9 match with Bragg planes of Hematite (202), (315) 228 respectively. The presence of a diffraction spot further confirms that the base material from which 229 Hematene is exfoliated has a Hematite crystallographic structure.

\subsection{How Hematene form?}


The mechanism of Hematene exfoliation and temporal control in understanding the surface energies

232 of most exposed crystallographic planes is shown in Figure 3. Note that, to understand the same,

233 first we have to know the surface energies of (104) planes in our finding and the naturally obtain 234 Hematite ores which have basal planes (0001) lying along the foliation plane [34]. Followed by 235 this, we will emphasize the hydroxylation of the Hematite surface. It takes a minute to an hour for 236 the hydroxylation, depending on the surface reactivity of each plane. It is a well-established fact 237 that the Hematite surface usually contains Fe atoms with an unoccupied state, acts as Lewis acids, 238 and reacts with Lewis bases such as hydroxyl ions via the available electron pair [33]. Due to the 239 same, hydroxyl ions adsorb on the surface of iron oxide via coordination with Fe atoms. The heat 240 treatment removes the surface hydroxyl group and forms water molecules due to the surface 241 condensation, and the same is desorbed later. The dehydroxylated Hematite nanoparticle on 242 exposure to ambient temperature forms three types of coordination based on the earlier study [30] 243 due to water vapour adsorbed by the (104) exposed facet. The hydroxylation of the Hematite surface 244 depends on the surface energies of exposed crystal facets. For instance, the surface energy of the 245 (104) plane of nanoparticles described here is around $2.64 \mathrm{~J} / \mathrm{m}^{2}$ [36], while that of (001) basal 246 planes in the case of naturally abundant Hematite ores are $1.53 \mathrm{~J} / \mathrm{m}^{2}$ [37]. So, the hydroxylation 247 time of both cases will differ and will finally affect the exfoliation step run by a secondary process 248 such as ultrasonication and solvent interaction with metal oxide surfaces. In this case, this 249 hydroxylation step is faster due to the more exposed (104) plane, leaving the exposed hydroxyl 250 group, which will further react with the absolute alcohol.

252 Next, the metal oxide is well known for the dehydration of alcohol and beta elimination reaction, 253 in which unsaturated organic compounds such as ethylene get produced according to the literature 254 described elsewhere [38]. Here, the alcohol hydrogen atom intercalates to the Fe-O-Fe linkage's 
oxygen, forming a hydroxyl moiety $(\mathrm{O}-\mathrm{H})$ covalent bond between two metal atoms. It takes place on (104) crystal facets leaving stress on the planes, which will finally lead to the formation of

257 Hematene sheets. It is bound initially by non-van der Waal forces. Besides this, this hydroxyl 258 moiety acts as a knob to control the magnetoelectric coupling in ferroelectrics such as $\mathrm{Ca}_{3} \mathrm{Mn}_{2} \mathrm{O}_{7}$.

259 It is also formed by hydrogen intercalation and, due to the same antiferromagnetic metal oxides, 260 shows ferrimagnetic properties [39]. It is also evident in the case of Hematene, which shows 261 ferrimagnetic behaviour, as stated above [23].

\section{3.4. Formation of Hematene Flakes}

263 So far, we have described the formation of a free-standing Hematene nanosheet. The free-standing 264 Hematene sheets are challenging to apply directly for device application. Nevertheless, to use them 265 as a functional material, we need a handful of systems where their influence can be observed 266 towards a specific functionality. In this regard, our goal is to use this type of $2 \mathrm{D}$ material for 267 photoelectrochemical water splitting applications. So, to achieve the same, as described above, we 268 ultrasonically transform Hematite nanoparticle film [Figure 1-III] into an exfoliated film with the 269 generation of flakes, which we called Hematene flakes by the 2D morphological characteristics as 270 observed by the FESEM. Here, Hematite film is sensitized by Hematene flakes after the 271 ultrasonication process. From here on, we will designate both Hematite films before and after

272 ultrasonication as B. S. and A. S. The homogenous solution mixture is used for thin-film coating, 273 as shown in Figure 1-I A, which on calcination at $500^{\circ} \mathrm{C}$ results in a Hematite film by following the 274 procedure described elsewhere [40].

\subsection{FESEM and EDX investigation of Hematene flakes sensitized thin film}


We have performed field emission scanning electron microscopy (FESEM) studies of Hematene

277 flakes to observed their morphology. It is followed by a detailed compositional characteristic of the

278 Hematene flakes to confirm the presence of $\mathrm{Fe}$ and $\mathrm{O}$ only, which composed the usual iron oxide 279 bulk counterpart. The morphology of calcined Hematite thin films (A.S. and B.S.), before and after 280 ultrasonication, has been studied, and their images are presented in Figures 4 A and B, respectively. 281 The FESEM image of the B.S. [Figure 4 A] obtained by the dip-coating methodology described in 282 our earlier study [41] shows anisotropic nanoparticles with the fusion of two nanoparticles in an 283 elongated pattern. The A.S. morphology is shown in the FESEM image [ Figure 4 B]. Here, the 284 particle morphology remains the same but the exfoliation results in more porous like features. In 285 B.S., particles have been uniformly deposited and possess a smooth surface, as confirmed from the 286 FESEM image of the thin film. While upon sonication, in figure $4 \mathrm{~B}$, the smooth surface was 287 converted to rough, as the particles from the film exfoliated.

288 Further, in Figure 4C, we have shown a FESEM image representing 2D flakes like structure formed 289 on the top of A.S. The flakes are also deposited freely on a coverslip [Inset; Figure 4B]. The purpose 290 is to understand that the $2 \mathrm{D}$ flakes like materials are originated from base Hematite nanoparticles. 291 Next, to determine the rough estimate of Hematene sheets and flakes thickness, 3D transformed 292 their corresponding HRTEM and FESEM images with Image J. The result obtained as shown in 293 Figure S4 reveals that the thickness is around $20 \mathrm{~nm}$ for the Hematene sheet after carefully looking 294 at the intensity scale bar of spectrum LUT images on the right side of the image. Similarly, the 295 thickness of Hematene flakes [Figure 4 D] is found to be $200 \mathrm{~nm}$.

296 Further, to rule out the presence of other elements or any external foreign objects such as dust 297 particles, we have made the EDX mapping and the line scan analysis of Hematene flakes. Here, the 298 EDX mapping [Figure 4 E] study confirms the distribution of Fe and O elements besides Sn, which mostly come from the substrate. Note that we have F- doped $\mathrm{SnO}_{2}$ as a substrate to deposit the 
Hematite film. The line scan analysis also confirms the presence of the same elements. From this observation, it is concluded that flakes like 2D materials are exfoliated due to the ultrasonication process. This structure is made of Hematene, as confirmed from the EDX mapping. It is also confirmed with the transparent nature of flakes, which shows the underneath FTO crystal. The usual Hematite SEM is devoid of this kind of morphology. The atomic \% wise distribution of elements $[\mathrm{Fe}=4 \%, \mathrm{O}=30 \%]$ confirms the stoichiometry of the Hematite. In this case, the formation of the hematite phase by XRD investigation directly confirms the $\alpha-\mathrm{Fe}_{2} \mathrm{O}_{3}$ stoichiometry (Hematite phase) of the materials described below.

Next, we have also carried out the AFM measurement of the Hematene sheet and flakes to determine their thicknesses. However, the result obtained [Figure S1] does not reveal the image found during the TEM and FESEM investigation. To determine the thickness of corresponding hematite nanoparticles, sheets and films, we have plotted the RMS roughness of the surface for each sample.

The result obtained is shown in Figure S2. For the Hematene sheet, the surface roughness decreases, while for Hematene flakes, it increases after the ultrasonication process. We have compared the FESEM of free-standing Hematene flakes (high magnification) with the one obtained on the thin film [Figure S 11] at a bulk level. Here, the surface roughness in both cases does not show any significant difference, and it appeared to be smooth on close inspection of FESEM images.

\subsection{Optical and Crystallographic properties of Hematene flakes and sheets}

318 We have measured the optical property of Hematene sheet, flakes and film before and after

319 ultrasonication (B.S. and A.S.) to understand light absorption characteristics and electronic properties with UV-Vis spectroscopy in both liquid and solid-state conditions. It is studied by the analysis of the absorption maxima $\lambda_{\max }$ shifting and energy bandgap $\left(\mathrm{E}_{\mathrm{g}}\right)$ calculation for indirect bandgap semiconductors with Tauc methodology. The understanding of Hematene flakes light 
absorption property is essential to decipher its role in the photoelectrochemical properties. For the 324 free-standing Hematene sheet, we have taken the exfoliated Hematite nanoparticle solution obtained 325 by the ultrasonication process. For the Hematene flakes, the exfoliated particles were used for UV326 Vis analysis by making an ethanol dispersion. Note that after exfoliation of thin film by 327 ultrasonication, we have obtained reddish-brown dispersion, which we believe the presence of 328 Hematene flakes and exfoliated nanoparticles from the film. The UV-Vis spectra of all samples, 329 including free-standing Hematene sheet, flakes, A.S. and B.S are shown in Figure 5 A. We have 330 observed the absorption intensity maintain its maxima $550 \mathrm{~nm}\left(\lambda_{\text {max }}\right)$ for both Hematene flakes and 331 Hematene sheet. The absorption spectra of both A.S. and B.S are shifted towards a shorter 332 wavelength $\lambda_{\max }=525 \mathrm{~nm}$.

333 In contrast, an additional absorption band appears at 400-450 $\mathrm{nm}$ in all cases, a characteristic 334 spectral signature of the Hematite UV-Vis spectrum, where two absorption bands at $400 \mathrm{~nm}$ and $335550 \mathrm{~nm}$ are typically observed [30]. Hematene flakes show the maximum absorption intensity of 336 all cases, making them a good absorber material for photoanode development. Next, we have seen 337 that its peak intensity decreases at $450 \mathrm{~nm}$ compared to the Hematene sheet, which still absorbs 338 photons below this range. It might be due to the concentration difference of the materials 339 understudied as the flakes like materials form diluted solution due to less density of it compared to 340 the nanosheet. In contrast, nanosheets are easily exfoliated into Ethanol solution and develop a 341 reasonably concentrated solution. However, a detailed electronic calculation understanding is 342 needed from the density functional theory to understand these sharp differences. It is also evident 343 that the light absorption intensity profile in the range from $550 \mathrm{~nm}-700 \mathrm{~nm}$ follows a different 344 trend than that of the B.S. and A.S. to be discussed next. It is an impressive result considering the 345 panchromatic light-harvesting ability of this particular Hematite based 2D material, a new finding 
to the best of our knowledge. It can be further extended in the interaction study of free-standing

347 Hematene sheets with light-harvesting proteins and pigments in hybrid organic optoelectronics.

348 Hematene flakes obtained in the ultrasonicated film mentioned above in the photoanode 349 functionality for light-harvesting are the primary evaluation criteria. It is found that the absorbance 350 intensity is more for the A.S. than B.S. [Figure 5 A]. Whether this increase in absorbance directly 351 influences Hematene flakes, we have plotted the UV-Vis absorbance spectrum of Hematene flakes 352 with that of A.S. and B.S., followed by a differential spectrum between A.S. and B.S. [Figure 5 B] 353 to understand it further. The differential spectrum shows the exact shape of the absorption spectrum 354 as that of Hematene flakes. The shape resembles the convoluted Hematene flakes absorbance 355 spectra, confirming its formation on the pristine Hematite film after the ultrasonication.

356 Whether the size of Hematene flakes or sheet has any influence on the spectral shape, we have 357 accordingly isolated three fractions of Hematene sheet suspension and measure their UV-Vis 358 spectra. It is shown in Figure S10. From the result obtained, it is evident that the shape of the 359 absorption spectrum does not change irrespective of the isolated fractions. It is concluded that the 360 spectral shape is independent of the size of the nanosheets present in the suspension.

361 We have plotted Tauc plots, as shown in Figure 5[C, E- H], of all samples for the indirect bandgap 362 of Hematite to know the effect of optical property changes on the electronic structure. Here, it is 363 evident that the Tauc profile for both Hematene sheet and flakes deviating from the standard pattern 364 of Tauc plots for B.S. and A.S. From here, it reveals that the energy bandgap ( $\left.\mathrm{E}_{\mathrm{g}}\right)$ of (Hematene 365 sheet $=2.6 \mathrm{eV} \&$ flakes $=2.4 \mathrm{eV}$ ) significantly differs from that of pristine Hematite. In comparison, 366 Hematene flakes sensitized film (A.S.) shows the usual band gap value of $2.1 \mathrm{eV}$. The Hematite has 367 an indirect phonon-assisted bandgap transition value of 1.9-2.2 eV [42]. The associated shift in 368 bandgap energy is attributed to the blue shift of the small Hematene nanostructures. Hematene 369 formation results in the rise of the conduction band and the lowering of the valance band, thereby 
resulting in a broader band gap value and blue shift of the absorption edge [43]. The same result

371 agrees with the band gap value of the Hematene obtained by the ultrasonication of natural Hematite 372 ores [22].

373 Next, we have presented the photoluminescence spectrum, as shown in Figure 5D. Here, on excitation with $320 \mathrm{~nm}$ wavelength, we have observed the emission spectra at $640 \mathrm{~nm}$. Hematite film A.S. and B.S. indicate that the emission peak shows a doublet pattern while the Hematene sheet shows singlet. The size of the iron oxide nanoparticle controls the changes in peak shape, a

377 common observation found in the X-ray emission spectra [44].

378 The scattering contribution and its quantitative analysis are also understood here to consider the 379 scattering contribution to optical properties. It is carried out by measuring the diffuse reflectance 380 spectrum of Hematite Film B.S. and A.S., followed by the Hematene sheet. Here we have applied the Kubelka -Munk function to the diffuse reflectance spectrum with the following equation:

$f(R)=\frac{(1-R)^{2}}{2 R}=\mathrm{k} / \mathrm{s}$

$\mathrm{R}$ is the diffuse reflectance of the layer relative to a standard such as magnesium oxide, $k$ is the molar absorption coefficient, and $s$ is the scattering coefficient of the sample. The diffuse reflectance spectra of all samples are shown in figure $6 \mathrm{~A}$. We have observed a similar type of diffuse reflectance pattern for both Hematite film A.S. and Hematene sheet while Hematite film B.S. shows high reflectance till $600 \mathrm{~nm}$, afterwards the reflectance lowers to $400 \mathrm{~nm}$. To understand the same, we have plotted the scattering coefficient $(s)$ and found that it varies significantly for pristine Hematite film, but for Hematite film A.S. and Hematene sheet, the pattern looks almost the same [Figure 6B]. It further confirms that the morphology of the Hematene sheet and flakes are the same as the scattering contribution, which is almost similar for both cases, whether film or dispersion. For pristine hematite film, the scattering gets higher from $550 \mathrm{~nm}$ onwards. Hence the 
absorbance changes due to different scattering coefficients for all samples. It is also found that the scattering and absorption coefficients of Hematite changes for morphology and size of the 396 nanoparticle [30].

397 Our previous study [40] shows that the forced hydrolysis of iron chloride salts in the presence of 398 Urea always results in maximum peak intensity for (104) Bragg planes, as confirmed by XRD. 399 Here, we have studied the detailed crystallographic properties of the Hematene flakes. We have 400 carried out the thin film XRD investigation of all three materials such as Hematene sheet, hematite 401 film B.S. and hematite film A.S. As shown in figure 6 C, we found that all the Bragg diffraction 402 peak shows the typical crystallographic structure of Hematite according to the JCPDS pattern. The 403 diffracted intensity of (110) plane decreases after the ultrasonication process for the Hematite Film 404 A.S. Next, on expanding the (104) and (110) peak [Figure 6D], it is evident that a new peak splitting 405 has been observed for (104) plane in case of Hematite film A.S. The (104) peak intensity after the 406 ultrasonication process decreases while a new peak appeared at $0.6^{\circ}$ from the original Bragg angle. 407 It further signifies the hydroxyl ion intercalation along the (104) peak described in the mechanism 408 [Figure 3]. A similar type of peak splitting is observed in the in-situ X-ray diffraction pattern of 409 chloroaluminate ion intercalation in Aluminium batteries [45].

\subsection{Surface properties of Hematene flakes and sheet from XPS study (X-Ray photoelectron}

411 spectroscopy)

412 The XPS (X-Ray photoelectron spectroscopy) is performed for hematite nanoparticle film obtained 413 from the heat treatment of rod-shaped particle, the hematite film A.S. and Hematene sheet 414 (dispersion) followed by a control measurement of substrate FTO (Fluorine doped tin oxide) on 415 which hematite film is deposited. The survey scan of all samples is shown in Figure S6. The 416 composition shows the variation in atomic ratio both before and after ultrasonication. 
417 The O1s core level spectrum of all samples is shown in Figure $7 \mathrm{~A}$. The first peak at $529 \mathrm{eV}$ comes 418 from the hematite lattice, while a second peak at $532 \mathrm{eV}$ is considered a defect peak, which is usual 419 of Hematene electronic structure as mentioned elsewhere [22]. The oxygen $1 \mathrm{~s}$ core level scan of 420 FTO reveals a peak at around $531.70 \mathrm{eV} \mathrm{[46],} \mathrm{and} \mathrm{this} \mathrm{peak} \mathrm{deviates} \mathrm{itself} \mathrm{from} \mathrm{the} \mathrm{defect} \mathrm{mediated}$ 421 peak. The Fe2p core level scan of hematite thin film B.S. and A.S. is shown in Figure 7B. Here, the 422 change in the oxidation state has not been observed after the ultrasonication process. Next, we have 423 fitted the oxygen 1 s core level spectrum with multiple peaks deconvolution [Figure $7 \mathrm{C}-\mathrm{F}$ ]. For 424 both hematite films, B.S. and A.S., the lattice peak, as mentioned above, comprises $\mathrm{O}^{2-}$ and O.H. 425 signatures followed by the formation of oxygen defect signature at $532 \mathrm{eV}$. Next, it is observed that 426 the intensity of this particular signature is more in hematite film A.S. than Hematite film B.S further 427 confirming the formation of Hematene flakes. Next, we have deconvoluted the O1S peak from the 428 Hematene sheet, signifying a pure defect-related peak at 532eV. Finally, fitting the O1S spectrum of FTO reveals a small spectral weight at $529 \mathrm{eV}$, pointing towards Hematite coating.

\subsection{Photoelectrochemical properties of Hematene flakes sensitized thin film}

431 We have applied Hematene flakes sensitized Hematite film for the photoelectrochemical response study due to its light absorption properties, as discussed above. We have not performed any measurement for the sheet-like Hematene materials as it is challenging to make a stable dispersion. There is a way to sensitize any photoanode materials with these Hematene materials like Graphene sensitized photoanode encountered in many studies. With this regard, Hematene coated titanium dioxide is published elsewhere as a sensitizing material [22]. Also, its application for the photoelectrochemical water splitting reaction is worth investigating due to the direct photoanode functionality of both bare Hematite and doped counterpart in terms of PEC water oxidation and matching of the valence band edge position with water oxidation potential [18]. Here, our goal is 
to see any difference in the photoelectrochemical response of this Hematene flakes sensitized 441 photoanode from that of the Hematite film. For the same, we have measured the photocurrent 442 density of both B.S. and A.S. in $1 \mathrm{M} \mathrm{KOH}$, as shown in Fig. 8A.

443 It depicts a comparison between the light and dark current densities for the B.S and A.S. In all 444 photocurrent density patterns, the usual photocurrent plateau is not observed, which mainly seen 445 for the nanostructured Hematite photoanode. In this case, the Hematite from which Hematene flakes are exfoliated is the direct conversion of Akageneite, crystal which usually results in more resistive action, while doped Hematite already well established to show higher photocurrent density [44]. Despite not having a Plateau-like feature, the photocurrent density of film A.S is higher than that of B.S. and displayed a photocurrent density of $50 \mu \mathrm{A} / \mathrm{cm}^{2}$. Here, the photocurrent density of bare Hematite film before sonication is very low in the $30 \mu \mathrm{A} / \mathrm{cm}^{2}$ at $0.6 \mathrm{~V}$. The slight increase in photocurrent density can be attributed to the more opening in the porosity of the photoanode materials (A.S.) due to the ultrasonication process. It is evident from the morphology discussion with the FESEM study, which shows a rougher surface for A.S. At the same time, the dark current density for A.S. is lower than that of B.S. Our earlier studies show that the Hematite films show considerable dark current density due to the presence of $\mathrm{Fe}^{2+}$ site, which on electrochemical oxidation result in extra electrons with a direct effect of Faradaic current density in the dark condition [41]. While in the A.S. film, as the ultrasonication process removes surface layers as exfoliated ones, the dark current density is low. The photocurrent density mentioned here is also not that high, but the ultrasonication process leads to a better photocurrent density, a new finding in this domain. We believe the direct role of Hematene flakes in the overall photocurrent density is due to the maximum absorbance shown by these flakes, followed by the A.S. film absorbance is higher than that of B.S. Besides these, Hematene also shows extra panchromatic light-harvesting above $550 \mathrm{~nm}$, and the additional increase in the photocurrent density is due to the collective action 
of the same. Besides these, the IPCE (incident photon to current conversion efficiency) of Hematene coated $\mathrm{TiO}_{2}$ photoelectrode shows increase efficiency in this region [22]. However, these ultrasonication methods will give rise to more efficient photoanodes, which are already well established. So, we keep it as the next objective for our future study with this new material.

\subsubsection{A qualitative understanding of the charge transfer process from the transient photocurrent}

\section{density}

Does the following question arise how Hematene flakes sensitized film (A.S.) shows its photoinduced charge transfer action compared to the Hematite film? The underlying physical phenomena for the same need to be understood. Here, we have carried out the transient photocurrent density study of films (A.S. and B.S.) under light ON-OFF configuration and results are shown in Figure $8 \mathrm{~B}$. If we consider the light $\mathrm{ON}$ part of the resultant data, the photocurrent density is considerably high for A.S. film than that of the B.S. Also, the spike spreads across both anodic and cathodic directions. The enhancement of both the anodic and cathodic spike intensity signifies bulk and excitonic surface recombination. If we carefully observe at $0.4 \mathrm{~V}$ [Figure $8 \mathrm{C}$ ], the intensity of this spike in the anodic direction is more than that of the cathodic direction.

In comparison, it is remaining the same for both cases for pristine Hematite film. If we consider the 2D Hematene nanosheet, the surface oxygen defect is validated by the XPS study as described above [Figure $7 \mathrm{C}$ and D]. Also, an operando synchrotron soft x-ray absorption spectroscopy study by Braun et al. reveals that the formation of the hole for the $\mathrm{O} 2 \mathrm{p}$ band or charge transfer band brings its maximum contribution photocurrent density at $0.4 \mathrm{~V}$ [47]. So, from here, we can justify that increase in the magnitude of spike formation in the anodic direction at $0.4 \mathrm{~V}$ is nothing but the function of the oxygen ligand present partially in 2D Hematene flakes along with the rest of oxygen ligands in the entire Hematite film after ultrasonication, which contributes to the formation of the 
hole and subsequent recombination. However, the experimental finding of the same is not done yet.

488 We here qualitatively propose a suggested physicochemical mechanism.

489 The photocurrent density reaches around $50 \mu \mathrm{A} / \mathrm{cm}^{2}$ at the light - ON mode, while it relaxes back 490 to the zero current during the light - OFF mode. The presence of a cathodic transient spike is due 491 to the bulk electron-hole recombination [48]. It is a direct first kind of study with Hematene. Next, 492 we have plotted the comparative limited chopped light current density profile of both films, as 493 shown in Figure 8 D. Here, limited stands for the slow speed of the shutter, which is embedded in 494 the light source. We have observed a different trend in the photocurrent relaxation phenomena for 495 exfoliated materials (A.S.) in both anodic and cathodic directions. Here, we see that current density 496 decayed in a delayed response. Before light-OFF mode, the photocurrent is excited for a while, 497 forming an additional spike until it reaches equilibrium. It falls again towards the cathodic direction. 498 Similarly, during the light-ON step, the photocurrent rises with a delayed intermediate response in 499 the cathodic regime before reaching the maximum magnitude in the anodic region. If we compare 500 the same with the film without ultrasonication (B.S.), we can also see a trend in the transient 501 photocurrent density.

502 The basic understanding and observation of a transient photocurrent spike need to be understood 503 regarding the photoinduced charge transfer effect. As described elsewhere [49, 50], the 504 photocurrent transient spike reaches a peak in the upward direction followed upon by decay to the 505 steady-state when light is turned on. After 10 seconds of photon illumination, if the light is turned 506 off, the zero current occurs and re-spiking when the light is turned back on again. The physical 507 significance of the falling photocurrent density is the conduction band electrons rapid removal into 508 the bulk, and spike formation occurs due to the recombination of electron holes by the surface state. 509 The sub- spike feature we discussed above is assigned as a sub-surface state generated peak. Now 510 coming back to our discussion about the transient photocurrent characteristic of A.S and B.S 
511 Hematite film, we have noticed that the light chopping with faster interval (figure $8 \mathrm{~B}$ ) does not 512 show this sub spike feature. Only when the light chopping speed is slow with longer intervals, this 513 specific feature is observed. The extra spike points towards slow removal of electrons, and in other 514 words, it shows back-reaction: the recombination of electrons and holes with sub-surface states as 515 a first glimpse, while a direct confirmation with different spectroscopic techniques is not available. 516 Finally, the extra spike is absent in other Hematite nanostructured films synthesized by an oleic 517 acid route [40]. It is evident when we compare the transient photocurrent density [Figure 8 E] of a 518 nano octahedron dispersed Hematite film [Figure $8 \mathrm{~F}$ ] with hematite film obtained by the water519 mediated route. The precursor chemistry leads to two different Hematite films with changes in the 520 surface electronic and atomic structure, as revealed by the extra spike-like feature. However, to get 521 detailed insight into the initial claim of surface and atomic structure changes, we need to do 522 synchrotron XRD and XAS, which is out of scope for the time being out of scope COVID-19 523 constraint. Our goal is to report here only the formation of Hematene sheets and flakes with their 524 photoelectrochemical properties. Also, the Hematene formation for oleic acid-derived Hematite 525 nano octahedron has not been studied. However, it will be the subject of further study.

\section{Conclusions}

527 The sonochemical treatment of hematite film leads to the formation of Hematene nanosheet and 528 flakes as supported by the detailed HRTEM investigation with the observation of both inward and 529 outward layers arrangement of atoms nanocrystallites. A thorough atomic structure understanding 530 with the d-spacing calculation reveals that the Hematene originated from the Hematite as it also 531 shows the crystallographically more exposed (104) plane. Based on the same, its formation mechanism is proposed and predictive that hydroxylation is faster due to more reactive surface due 533 to the high surface energy of (104) planes. The XPS study validates the usual oxygen defect 534 signature of Hematene at $532 \mathrm{eV}$, which further confirms the enhanced photoelectrochemical 
535 functionality of the Hematite film after ultrasonication treatment and the formation of the sub536 surface state. The photocurrent density increased up to $50 \mu \mathrm{A} / \mathrm{cm}^{2}$ for Hematene flakes sensitized 537 Hematite photoanode due to its enhanced light-harvesting action, while the pristine shows current 538 density up to $30 \mu \mathrm{A} / \mathrm{cm}^{2}$. Though it is not a fantastic performance, the future outlook of such 539 sonochemical modification and making of Hematene flakes on champion photoanode will boost its 540 efficiency further for the solar water-splitting process.

\section{Supporting Information}

542 Electronic Supplementary Information (ESI) is available: AFM data statistics, FESEM images, Size 543 distribution, XPS survey scan, Fe2p core level scan and fitted spectrum, UV-vis spectrum of Hematene 544 extracts.

\section{Conflicts of interest}

546 There are no conflicts to declare.

\section{Acknowledgements}

548 The author (D.K.B) acknowledge the funding received from the J.U. management start-up grant: 54911 (39)/17/004/2017SG to carry out this work. The Nano Mission Project "SR/NM/NS-20/2014" 550 supported the FESEM and XRD facilities used in this study. Next, We want to acknowledge the 551 TEM facility of the Center for Nano and Soft Matter Sciences (CeNS), Bangalore, for the HRTEM 552 characterization, followed by the Central Research Facility (CRF) U.V.- Vis DRS, Thin Film XRD, 553 Atomic Force Microscopy and Fluorescence measurement. For the XPS measurement, the Central 554 Surface Analytical Facility of IIT Bombay is duly acknowledged here. 


\section{References}

1. Novoselov, K. S.; Gei, A. K.; Morozov, S. V.; Jiang, D. Electric Field Effect in Atomically Thin Carbon Films. Science 2004, 306, 666-669.

2. Nicolosi, V.; Chhowalla, M.; Kanatzidis, M. G.; Strano, M. S.; Coleman, J. N. Liquid exfoliation of layered materials. Science 2013, 340, 1226419.

3. Naguib, M.; Mochalin, V. N.; Barsoum, M. W.; Gogotsi, Y. 25th anniversary article: MXenes: a new family of two-dimensional materials. Adv. Mater. 2014, 26(7), 992-1005.

4. Akhtar, M.; Anderson, G.; Zhao, R.; Alruqi, A.; Mroczkowska, J. E.; Sumanasekera, G., Jasinski, J. B. Recent advances in synthesis, properties, and applications of phosphorene. NPJ 2D Mater Appl. 2017, $1(1), 1-13$.

5. Mannix, A. J.; Kiraly, B.; Hersam, M. C.; Guisinger, N. P. Synthesis and chemistry of elemental 2D materials. Nat. Rev. Chem. 2017, 1(2), 1-14.

6. Butler, S. Z.; Hollen, S. M.; Cao, L.; Cui, Y.; Gupta, J. A.; Gutiérrez, H. R.; Heinz, T. F.; Hong, S. S.; Huang, J.; Ismach, A. F.; Johnston-Halperin, E.; Kuno M.; Plashnitsa, V. V.; Robinson, R. D.; Ruoff, R. S.; Salahuddin, S.; Shan, J.; Shi, L.; Spencer, M. G.; Terrones, M.; Windl, W.; Goldberger, J. E. Progress, challenges, and opportunities in two-dimensional materials beyond Graphene. ACS nano 2013, 7(4), 2898-2926.

7. Xiao, X.; Song, H.; Lin, S.; Zhou, Y.; Zhan, X.; Hu, Z.; Zhang, Q.; Sun, J.; Yang, B.; Li, T.; Jiao, L. Scalable salt-templated synthesis of two-dimensional transition metal oxides. Nat. Comm. 2016, 7(1), $1-8$.

8. Xu, M.; Liang, T.; Shi, M.; Chen, H. Graphene-like two-dimensional materials. Chem. Rev. 2013, 113(5), 3766-3798.

9. Ma, Y.; Li, B.; Yang, S.; Ultrathin two-dimensional metallic nanomaterials. Mater. Chem. Front., 2018, 2(3), 456-467.

10. Xu, T.; Zhang, J.; Zhu, Y.; Wang, J.; Shimada, T.; Kitamura, T.; Zhang, T.Y. Two-dimensional polar metal of a PbTe monolayer by electrostatic doping. Nanosc. Horiz. 2020, 5(10),1400-1406.

11. Neto, A.C.; Guinea, F.; Peres, N.M.; Novoselov, K.S.; Geim, A.K. The electronic properties of Graphene. Rev. Mod. Phy. 2009, 81(1),109-162.

12. Sun, Z.; Liao, T.; Dou, Y.; Hwang, S.M.; Park, M.S.; Jiang, L.; Kim, J.H.; Dou, S.X. Generalized selfassembly of scalable two-dimensional transition metal oxide nanosheets. Nat. Comm. 2014, 5(1), 1-9. 
13. Montgomery, M.J.; Sugak, N.V.; Yang, K.R.; Rogers, J.M.; Kube, S.A.; Ratinov, A.C.; Schroers, J.; Batista, V.S.; Pfefferle, L.D. Semiconductor-to-conductor transition in 2D copper (II) oxide nanosheets through surface sulfur-functionalization. Nanoscale 2020, 12(27), 14549-14559.

14. Mishra, M.; Chun, D.M. $\alpha-\mathrm{Fe}_{2} \mathrm{O}_{3}$ as a photocatalytic material: A review. Appl Catal. A: Gen. 2015, 498,126-141.

15. Zeng, H.; Li, J.; Liu, J.P.; Wang, Z.L.; Sun, S. Exchange-coupled nanocomposite magnets by nanoparticle self-assembly. Nature 2002, 420(6914), 395-398.

16. Chen, J.; Xu, L.; Li, W.; Gou, X. $\alpha-\mathrm{Fe}_{2} \mathrm{O}_{3}$ nanotubes in gas sensor and lithium-ion battery applications. Adv. Mater. 2005, 17(5), 582-586.

17. Bora, D.K.; Deb, P. Fatty acid binding domain mediated conjugation of ultrafine magnetic nanoparticles with albumin protein. Nanosc. Res. Lett. 2009, 4(2),138-143.

18. Bora, D.K.; Braun, A.; Constable, E.C. "In rust we trust". Hematite-the prospective inorganic backbone for artificial photosynthesis. Energ. Environ. Sci. 2013, 6(2), 407-425.

19. Gong, M.; Li, Y.; Wang, H.; Liang, Y.; Wu, J.Z.; Zhou, J.; Wang, J.; Regier, T.; Wei, F.; Dai, H. An advanced $\mathrm{Ni}-\mathrm{Fe}$ layered double hydroxide electrocatalyst for water oxidation. J. Am. Chem. Soc. 2013, 135(23), 8452-8455.

20. Bang, J.H.; Suslick, K.S. Applications of ultrasound to the synthesis of nanostructured materials. Adv. Mater. 2010, 22(10),1039-1059.

21. James, D. K.; Tour, J. M. Graphene: powder, flakes, ribbons, and sheets. Acc. Chem. Res. 2013, 46(10), 2307-2318.

22. Balan, A.P.; Radhakrishnan, S.; Woellner, C.F.; Sinha, S.K.; Deng, L.; de Los Reyes C.; Rao, B.M.; Paulose, M.; Neupane, R.; Apte, A.; Kochat, V. Exfoliation of a non-van der Waals material from iron ore hematite. Nat. Nanotech. 2018, 13(7), 602-609.

23. Gonzalez, R. I.; Mella, J.; Díaz, P.; Allende, S.; Vogel, E. E.; Cardenas, C.; Munoz, F. Hematene: a 2D magnetic material in van der Waals or non-van der Waals heterostructures. 2D Mater., 2019, 6(4), 045002.

24. Bandyopadhyay, A.; Frey, N.C.; Jariwala, D.; Shenoy, V.B. Engineering magnetic phases in twodimensional non-van der Waals transition-metal oxides. Nano Lett., 2019, 19(11), 7793-7800.

25. Chen, D.; Zhang, G.; Sun, W.; Li, J.; Cheng, Z.; Wang, Y. Tuning the magnetism of two-dimensional hematene by ferroelectric polarization. Phys. Chem. Chem. Phys. 2019, 21(23), 12301-12309.

26. Zhang, Z.; Vieira, D.; Barralet, J.E.; Merle G. 2D hematene, a bioresorbable electrocatalytic support for glucose oxidation. 2D Mater. 2020, 7(2), 025044. 
27. Mohanty, B.; Wei, Y.; Ghorbani-Asl, M.; Krasheninnikov, A.V.; Rajput, P.; Jena, B.K.; Revealing the defect-dominated oxygen evolution activity of hematene. J. Mater. Chem. A, 2020, 8(14), 6709-6716.

28. Mahmood, N.; De Castro, I.A.; Pramoda, K.; Khoshmanesh, K.; Bhargava, S.K. Kalantar-Zadeh, K.; Atomically thin two-dimensional metal oxide nanosheets and their heterostructures for energy storage. Energ. Stor. Mater. 2019, 16, 455-480.

29. Patra, A.K.; Kundu, S.K.; Bhaumik, A.; Kim, D. Morphology evolution of single-crystalline hematite nanocrystals: magnetically recoverable nanocatalysts for enhanced facet-driven photoredox activity. Nanoscale, 2016, 8(1), 365-377.

30. Cornell, R.M.; Schwertmann, U. The iron oxides: structure, properties, reactions, occurrences and uses. John Wiley \& Sons, 2003.

31. Zhou, S.; Li, J.; Gilroy, K.D.; Tao, J.; Zhu C.; Yang, X.; Sun, X.; Xia, Y. Facile synthesis of silver nanocubes with sharp corners and edges in an aqueous solution. ACS Nano, 2016, 10(11), 9861-9870.

32. Ghorai, A.; Ray, S. K.; Midya, A.; Ethylenediamine-Assisted high yield exfoliation of $\mathrm{MoS}_{2}$ for flexible solid-state supercapacitor application. ACS Appl. Nano Mater. 2019, 2(3), 1170-1177.

33. Bailey, J. K.; Brinker, C. J.; Mecartney, M. L. Growth mechanisms of iron oxide particles of differing morphologies from the forced hydrolysis of ferric chloride solutions. J. Col. Interf. Sci, 1993, 157(1), 1-13.

34. Song, Y.; Liu, T.; Li, M.; Yao, B.; Kou, T.; Feng, D.; Wang, F.; Tong, Y.; Liu, X.X.; Li, Y. Engineering of mesoscale pores in balancing mass loading and rate capability of hematite films for electrochemical capacitors. Adv. Energ. Mater. 2018, 8(26), 1801784.

35. Fan, H.; Song, B.; Liu, J.; Yang, Z.; Li, Q. Thermal formation mechanism and size control of spherical hematite nanoparticles, Mater. Chem. Phys. 2005, 89, 321-325.

36. Barbosa, P.F.; Lagoeiro, L. Crystallographic texture of the magnetite-hematite transformation: Evidence for topotactic relationships in natural samples from Quadrilátero Ferrífero, Brazil. Am. Mineral. 2010, 95(1),118-125.

37. Zhao, J.; Huggins, F.E.; Feng, Z.; Huffman, G.P. Ferrihydrite: surface structure and its effects on phase transformation. Clay. Clay Miner., 1994, 42(6), 737-746.

38. Reeves, N. J.; Mann, S.; Influence of inorganic and organic additives on the tailored synthesis of iron oxides. J. Chem. Soc. Faraday T, 1991, 87(24), 3875-3880.

39. Mackrodt. W.C.; Atomistic simulation of oxide surfaces. Phys. Chem. Miner. 1988, 15(3), 228-237. 
40. Shinohara, Y.; Satozono, H.; Nakajima, T.; Suzuki, S.; Mishima, S. Study of the Interaction of Ethanol with the Bronsted and Lewis Acid Sites on Metal Oxide Surfaces Using the DV-X. ALPHA. Method. J. Chem. Softw. 1998, 4, 41-50.

41. Ni, J. Y.; Wang, P. S.; Lu, L. J.; Xiang, J. H. Realizing Magnetoelectric Coupling with Hydrogen Intercalation. Phys. Rev. Lett. 2019, 122(11), 117601.

42. Agarwal, P.; Bora, D. K.; The Photoelectrochemical Response of a $\beta$-Carotene Coated Hematite Nanoarchitecture Biophotoelectrode with Cauliflower and Nano Octahedron Types Morphologies. $J$. Electrochem. Soc., 2019, 166(12), H541-H548.

43. Bora, D. K.; Braun, A.; Erni, R.; Fortunato, G.; Graule, T.; Constable, E. C. Hydrothermal treatment of a hematite film leads to highly oriented faceted nanostructures with enhanced photocurrents. Chem. Mater., 2011, 23(8), 2051-2061.

44. Kennedy, J. H.; Frese Jr., K. W.; Photooxidation of water at $\alpha-\mathrm{Fe}_{2} \mathrm{O}_{3}$ electrodes. J. Electrochem. Soc. 1978, 125(5), 709-714.

45. He, Y. P.; Miao, Y. M.; Li, C. R.; Wang, S. Q.; Cao, L.; Xie, S. S.; Yang, G. Z.; Zou, B. S.; Burda, C. Size and structure effect on optical transitions of iron oxide nanocrystals. Phys. Rev. B, 2005, 71(12), 125411.

46. Gilbert, B.; Katz, J. E.; Denlinger, J. D.; Yin, Y.; Falcone, R.; Waychunas, G. A. Soft X-ray spectroscopy study of the electronic structure of oxidized and partially oxidized magnetite nanoparticles. J. Phys. Chem. C, 2010, 114(50), 21994-22001.

47. Pan, C.J.; Yuan, C.; Zhu, G.; Zhang, Q.; Huang, C.J.; Lin, M.C.; Angell, M.; Hwang, B.J.; Kaghazchi, P.; Dai, H. An operando X-ray diffraction study of chloroaluminate anion-graphite intercalation in aluminum batteries. Proc. Nat. Acad. Sci. 2018, 115(22), 5670-5675.

48. Swallow, J.E.; Williamson, B.A.; Whittles, T.J.; Birkett, M.; Featherstone, T.J.; Peng, N.; Abbott, A.; Farnworth, M.; Cheetham, K.J.; Warren, P.; Scanlon, D.O. Self-compensation in transparent conducting F-doped $\mathrm{SnO}_{2}$. Adv. Func. Mater., 2018, 28(4), 1701900.

49. Braun, A.; Sivula, K.; Bora, D. K.; Zhu, J.; Zhang, L.; Gratzel, M.; Guo, J.; Constable, E. C.; Direct observation of two-electron holes in a hematite photoanode during photoelectrochemical water splitting. J. Phys. Chem. C, 2012, 116(32), 16870-16875.

50. Dotan, H.; Sivula, K.; Grätzel, M.; Rothschild, A.; Warren, S. C. Probing the photoelectrochemical properties of Hematite $\left(\alpha-\mathrm{Fe}_{2} \mathrm{O}_{3}\right)$ electrodes using hydrogen peroxide as a hole scavenger. Energ. Environ. Sci. 2011, 4(3), 958-964. 
679 51. Bora, D. K.; Rozhkova, E. A.; Schrantz, K.; Wyss, P. P.; Braun, A.; Graule, T.; Constable, E. C.; 680 Functionalization of Nanostructured Hematite Thin-Film Electrodes with the Light-Harvesting 681 Membrane Protein C-Phycocyanin Yields an Enhanced Photocurrent. Adv. Func. Mater. 2012, 22(3), $682490-502$.

683 52. Eggleston, C. M.; Shankle, A. J.; Moyer, A. J.; Cesar, I.; Grätzel, M.; Anisotropic photocatalytic 684 properties of Hematite. Aq. Sci. 2009, 71(2), 151-159. 